\section{A) Check for updates}

Cite this: Mater. Adv., 2020, 1,1186

Received 29th April 2020 Accepted 22nd June 2020 DOI: 10.1039/d0ma00258e

rsc.li/materials-advances

\title{
Bond states, moiré patterns, and bandgap modulation of two-dimensional BN/SiC van der Waals heterostructures $\dagger$
}

\author{
Maolin Bo, (D) * Hanze Li, Anlin Deng, Lei Li, Chuang Yao, (D) Zhongkai Huang and \\ Cheng Peng (D) *
}

\begin{abstract}
The matched lattice strain of the graphene/hexagonal boron nitride (BN) heterojunction that is less than $2 \%$ is known to form a moire pattern. However, in the BN/SiC heterojunction, the formation of a moiré pattern for a lattice strain more than $5 \%$ is a novel phenomenon. This study aims to determine moiré patterns on the $\mathrm{BN} / \mathrm{SiC}$ heterojunction at lattice strains of $5 \%$ and $7 \%$ and different incident angles, which can be applied to photoelectric detection technology. The BN/SiC-1 and BN/SiC-2 heterojunctions formed in this study had bandgaps of 0.851 and $1.373 \mathrm{eV}$, respectively. Furthermore, we observed that when two BN/SiC heterojunctions have indirect bandgaps, the BN/SiC van der Waals heterojunction can be a potential photoelectric material. We analyze the bonding states using the bond-charge model and calculate the potential functions of the antibonding, bonding, and nonbonding states. This study provides a theoretical reference for the precise regulation of the charge density and chemical bonding states of the 2D material surface.
\end{abstract}

\section{Introduction}

The successful preparation of a two-dimensional (2D) atomic layer of the planar graphene structure has encouraged researchers to investigate $2 \mathrm{D}$ nanomaterials..$^{1-4}$ Owing to the similarities between the structures and bonding properties of hexagonal boron nitride (BN) and graphite, shortly after the preparation of graphene, a single layer of hexagonal honeycomb BN was successfully prepared by micromechanical stripping. ${ }^{5} \mathrm{BN}$ has a layered hexagonal honeycomb structure with a wide band gap of $4.6 \mathrm{eV}{ }^{6}$ However, to increase the efficiency of light absorption and convert the $2 \mathrm{D}$ hexagonal $\mathrm{BN}$ sheet to a highly efficient photocatalytic material, the band gap of $\mathrm{BN}$ needs to be reduced to approximately $1.5 \mathrm{eV}{ }^{7} \mathrm{Hu}$ et al. found that the band gap can be reduced through lattice strain regulation of the BN sheet. ${ }^{8}$ Tang et al. found that band gap reduction can be achieved through electric field regulation of the $\mathrm{BN}$ sheet. ${ }^{9} \mathrm{He}$ et al. achieved the regulation of electronic structures in two-dimensional transition metaldoped BN materials through hydrogen and fluorine chemical modification. ${ }^{10}$ These studies provide the theoretical references for band gap regulation.

Chongqing Key Laboratory of Extraordinary Bond Engineering and Advanced Materials Technology (EBEAM), Yangtze Normal University, Chongqing 408100, China.E-mail:bmlwd@yznu.edu.cn,20090008@yznu.cn

$\dagger$ Electronic supplementary information (ESI) available. See DOI: 10.1039/ d0ma00258e
When manufacturing electronic devices, the majority of the 2D materials are grown on metals or semiconductor substrates, which causes strain in the crystal lattice. ${ }^{11-13}$ There exists a difference of $1.84 \%$ between the lattice constants of graphene and hexagonal BN. ${ }^{14}$ Additionally, the graphene/hexagonal BN system forms a moiré pattern. ${ }^{15,16}$ Furthermore, Kang et al. studied the 2D MoS$/ 2 / \mathrm{MoSe}_{2}$ heterojunction and found that the strength of the van der Waals interaction between the $\mathrm{MoS}_{2}$ and $\mathrm{MoSe}_{2}$ layers is not sufficient to eliminate this mismatch resulting in lattice strain. Zhang et al. studied the interlayer couplings, moiré patterns, and 2D electronic superlattices in $\mathrm{MoS}_{2} / \mathrm{WSe}_{2}$ hetero-bilayers. ${ }^{17}$ Rosenberger et al. studied the moiré pattern of a transition metal dichalcogenide heterostructure and found that the angle rotation is related. ${ }^{18} \mathrm{CaO}$ et al. found that the superconducting properties of two layers of graphene were exhibited at specific angles (approximately $1.1^{\circ}$ ). ${ }^{19}$ Therefore, matching lattice strains of interlayer couplings and angle rotation of the two atomic layer heterojunctions will form a moire pattern. ${ }^{20}$ The formation of the moiré pattern has led to the emergence of many novel physical and chemical properties. ${ }^{21-24}$

In this study, we found that lattice strains of $5 \%$ and $7 \%$ will cause a moiré pattern on the $\mathrm{BN} / \mathrm{SiC}$ heterojunctions. The band gaps of $\mathrm{BN} / \mathrm{SiC}-1$ and -2 heterojunctions are approximately 0.851 and $1.373 \mathrm{eV}$, respectively. Furthermore, these heterostructures effectively modulate the bandgaps of BN (4.661 eV) and $\mathrm{SiC}(2.108 \mathrm{eV})$. This provides a basis for designing $2 \mathrm{D}$ semiconductor heterojunction materials, where the lattice 
strain can be controlled in different directions to regulate the band gap. Furthermore, we calculate the potential of the antibonding, bonding, and nonbonding functions using the bond-charge (BC) model, which provides a theoretical reference for the precise regulation of the charge density and chemical bonding states of the 2D material surface.

\section{Principles}

\subsection{DFT calculations}

The two-dimensional material geometry structure was obtained from the crystal Material Projects database. Then, through the interface function of the QuantumWise's software, structural models of the heterojunction $(\sim 30-50$ kinds of possible structures) are established, and the lattice strain value that matches the geometry structure from the database is the obtained lattice strain. The model of the initial structure only considers the lattice strain of the $\mathrm{BN}$ atomic layer. The average strain in the BN layer is listed in Table 1. The initial structure is geometrically optimized in the Vienna ab initio simulation package, and the relaxation model includes optimization of atomic positions and unit cells. We set the energy cut off of the plane wave at $400 \mathrm{eV}$ and the Brillouin zone at $4 \times 4 \times 4 k$-points. To acquire the optimized geometric structures, the energy convergence criterion is $10^{-5} \mathrm{eV}$, and the atomic force convergence is $0.01 \mathrm{eV} \AA^{-1}$. Next, the Cambridge Sequential Total Energy Package (CASTEP) was used to optimize the geometry and increase the accuracy of calculating chemical bonding and electronic properties.

The DFT calculations, using the norm-conserving potentials of the hybrid density functional, were implemented in the CASTEP software. We use the Heyd-Scuseria-Ernzerhof (HSE06) ${ }^{25}$ hybrid density functional to describe the electron exchange and correlation potential. There are no constraints on the atomic layer. Also, we consider non self-consistent dipole correction in the calculation process. The thickness of the vacuum region is $14 \AA$. The energy convergence criterion is set to $1 \times 10^{-6} \mathrm{eV}$, and the plane wave cut-off energy is set to $700 \mathrm{eV}$. To consider the long-range van der Waals interaction, we use the TS scheme for DFT-D calculation. Table 1 summarizes the parameters used in the DFT calculations.

Table 1 (a) Angles and lattice parameters of the $\mathrm{BN} / \mathrm{SiC}$ heterostructures. (b) Corresponding set values of $k$-points, cut off, and lattice strain $\varepsilon$ of the $\mathrm{BN} / \mathrm{SiC}$ heterostructures

(a)

\begin{tabular}{llllllll}
\hline & \multicolumn{3}{l}{ Angles } & & \multicolumn{3}{c}{ Lattice parameters } \\
\cline { 2 - 4 } Structures & $\alpha\left(^{\circ}\right)$ & $\beta\left({ }^{\circ}\right)$ & $\gamma\left({ }^{\circ}\right)$ & & $A(\AA)$ & $B(\AA)$ & $C(\AA)$ \\
\hline BN/SiC-1 & 90 & 90 & 90 & & 5.36 & 6.19 & 24.93 \\
BN/SiC-2 & 90 & 90 & 109.11 & 5.36 & 8.19 & 25.93 \\
\hline
\end{tabular}

(b)

\begin{tabular}{llllrrr}
\hline & $k$-points & Cut off $(\mathrm{eV})$ & $\varepsilon_{11}(\%)$ & $\varepsilon_{22}(\%)$ & $\varepsilon_{12}(\%)$ & $\varepsilon(\%)$ \\
\hline $\mathrm{BN} / \mathrm{SiC}-1$ & $10 \times 9 \times 2$ & 700 & 6.65 & -5.20 & 10.26 & 7.37 \\
$\mathrm{BN} / \mathrm{SiC}-2$ & $10 \times 6 \times 2$ & 700 & 6.65 & -11.12 & 0.00 & 5.93
\end{tabular}

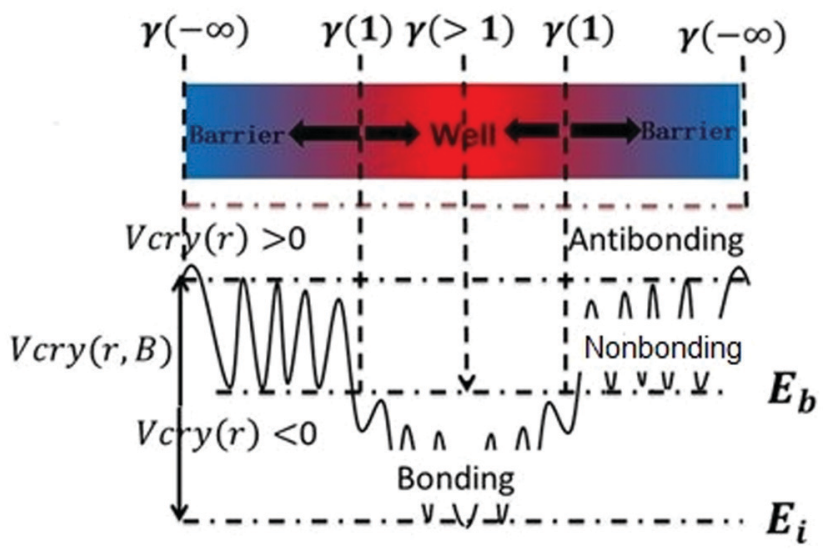

Fig. 1 Schematic of the bond-charge (BC) model in combination with the BOLS notation

\subsection{BOLS-BC model}

The following equations are used to describe the electronic state of a specific chemical bond, based on the bond-orderlength-strength (BOLS) notation: ${ }^{26}$

$$
\begin{aligned}
\frac{E_{i}}{E_{\mathrm{B}}} & =\left(\frac{1 / d_{i}}{1 / d_{\mathrm{B}}}\right)^{\mathrm{m}} \propto \frac{V_{\text {cry }}\left(r_{i}\right)}{V_{\text {cry }}\left(r_{\mathrm{B}}\right)} \\
& =\gamma ;\left\{\begin{array}{l}
\gamma>1, \text { deepening the potential well } \\
\gamma<1, \text { strengthening the potential energy barrier }
\end{array}\right.
\end{aligned}
$$
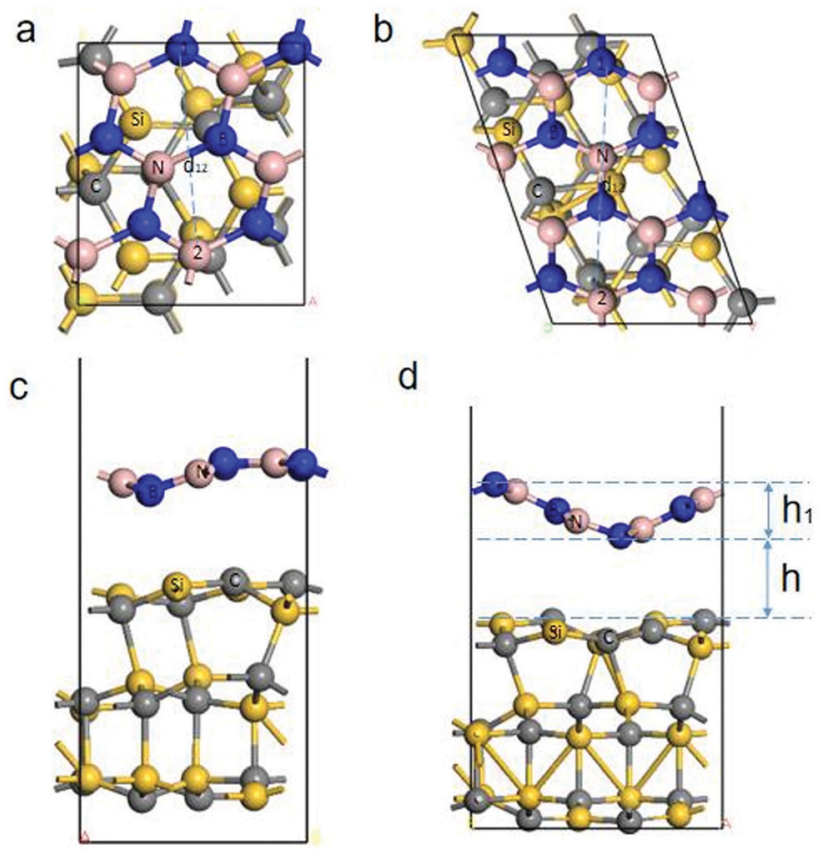

Fig. 2 (a) and (b) Top views of the BN/SiC-1 and 2 heterostructures. (c) and (d) Side views of the BN/SiC-1 and 2 heterostructures. The height (h) between the $\mathrm{BN}$ and $\mathrm{SiC}$ layers is the distance between the $\mathrm{BN}$ and $\mathrm{SiC}$ layers in the thickness direction of the slab. The height $h_{1}$ is the height of the wave shape structure of heterostructures 1 and 2 . The distance $d_{12}$ is the distance between atom 1 and atom 2 of heterostructures 1 and 2 . 
Table 2 The formation energy $E_{\text {form }}=E_{\text {total }}^{\text {heterostructure }}-E_{\text {total }}^{\mathrm{SiC}}-E_{\text {total }}^{\mathrm{BN}}$ of $\mathrm{BN} / \mathrm{SiC}-1$ and $\mathrm{BN} / \mathrm{SiC}-2$ heterostructures

\begin{tabular}{lllllll}
\hline$E_{\text {total }}^{\text {heterostructure }}$ & $(\mathrm{eV})$ & \multicolumn{2}{l}{$E_{\text {total }}^{\mathrm{SiC}}(\mathrm{eV})$} & \multicolumn{2}{l}{$E_{\text {total }}^{\mathrm{BN}}(\mathrm{eV})$} & \multicolumn{2}{l}{$E_{\text {form }}(\mathrm{eV})$} \\
\hline $\mathrm{BN} / \mathrm{SiC}-1$ & -5232.97 & $\mathrm{SiC}$ & -3142.01 & $\mathrm{BN}$ & -2089.70 & -1.26 \\
$\mathrm{BN} / \mathrm{SiC}-2$ & -6720.69 & $\mathrm{SiC}$ & -3927.88 & $\mathrm{BN}$ & -2791.65 & -1.16
\end{tabular}

Table 3 Work function, band gap, layer spacing $h$ and $h_{1}$ of BN/SiC structures

\begin{tabular}{lllll}
\hline & $\begin{array}{l}\text { Work function } \\
(\mathrm{eV})\end{array}$ & $\begin{array}{l}\text { Band } \\
\text { gap }(\mathrm{eV})\end{array}$ & $\begin{array}{l}\text { Layer } \\
\text { spacing } h(\AA)\end{array}$ & $\begin{array}{l}\text { Layer } \\
\text { spacing } h_{1}(\AA)\end{array}$ \\
\hline $\mathrm{BN} / \mathrm{SiC}-1$ & 4.512 & 0.851 & 2.43 & 0.83 \\
$\mathrm{BN} / \mathrm{SiC}-2$ & 4.912 & 1.373 & 2.73 & 1.60
\end{tabular}

Table 4 Initial height $(H)$, relaxation height $(h)$, and distance between atom 1 and atom 2 for different $\mathrm{BN} / \mathrm{SiC}$ heterostructures

\begin{tabular}{llll}
\hline & Initial height $(H)$ & Relaxation height $(h)(\AA)$ & $d_{12}(\AA)$ \\
\hline BN/SiC-1 & 3.20 & 2.43 & 4.092 \\
BN/SiC-1 & 2.99 & 2.42 & 4.092 \\
BN/SiC-1 & 2.79 & 2.41 & 4.090 \\
BN/SiC-1 & 2.59 & 2.47 & 4.091 \\
BN/SiC-1 & 2.39 & 2.36 & 4.089 \\
BN/SiC-2 & 3.52 & 2.73 & 5.844 \\
BN/SiC-2 & 3.32 & 2.79 & 5.825 \\
BN/SiC-2 & 3.12 & 2.76 & 5.859 \\
BN/SiC-2 & 2.92 & 2.71 & 5.840 \\
BN/SiC-2 & 2.72 & 2.67 & 5.836
\end{tabular}

where $d_{i}$ is the bond length of the atom, $E_{i}$ represents the single bond energy, $V_{\text {cry }}\left(r_{i}\right)$ is the crystal potential, $m$ is the bond nature, $B$ represents bulk atoms, and $i$ counts from the outermost atomic layer inward up to the third indicator layer $(i \leq 3)$.

Eqn (1) describes the relationship between the chemical bonds (energy and length) and crystal potential functions based on the BOLS notation. ${ }^{27}$ The potential function $\gamma V_{\text {cry }}\left(r_{i}\right)$ may become deeper $(\gamma>1$ for potential well formation) or shallower $(\gamma<1$ for potential barrier formation) than the corresponding $V_{\text {cry }}\left(r_{\mathrm{B}}\right)$ of the specific constituent. ${ }^{28}$

$$
\left\{\begin{array}{l}
V_{\text {cry }}\left(r_{i}\right)=\left(\frac{1}{4 \pi \varepsilon_{0}}\right) \iiint \mathrm{d}^{3} r_{i} \iiint \mathrm{d}^{3} r_{i} \frac{\Delta \rho_{\text {hole }}(r) \Delta \rho_{\text {electron }}(r)}{r_{i}} \\
E_{\mathrm{G}}=2\left|V_{\mathrm{l}}\right| \propto E_{i} \\
V_{1}=\int V_{\text {cry }}\left(r_{i}\right) \mathrm{e}^{i k r} \mathrm{~d} r
\end{array}\right.
$$

Eqn (2) describes the relationship between the bond energy $E_{i}$, crystal potential $V_{\text {cry }}\left(r_{i}\right)$, bandgap $E_{\mathrm{G}}$, and the deformation charge density $\Delta \rho\left(r_{i}\right)$. Fig. 1 illustrates the BC model with the BOLS notation.

\section{Results and discussion}

\subsection{Geometry structure}

The geometry structure of the different strains is selected based on QuantumWise's interface modeling, and the geometry structure to be optimized is obtained. The lattice strain $(>5 \%)$ produces the $\mathrm{BN} / \mathrm{SiC}$ heterostructures (orthorhombic (Fig. 2a and c) or rhombohedral (Fig. 2b and d)). Fig. 2c and d show that both $\mathrm{BN} / \mathrm{SiC}-1$ and $\mathrm{BN} / \mathrm{SiC}-2$ heterojunctions form a a

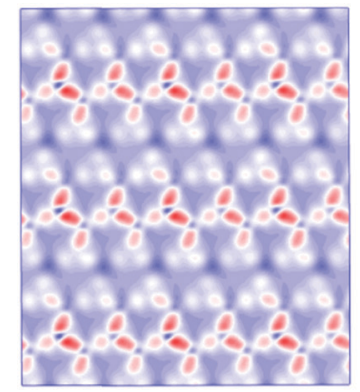

d

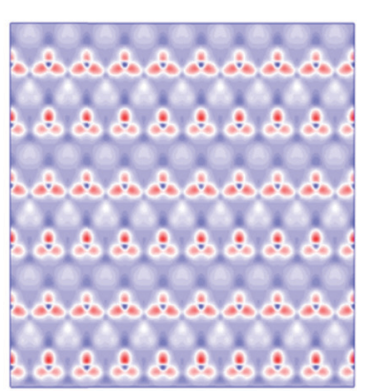

$\theta=0^{\circ}$ b

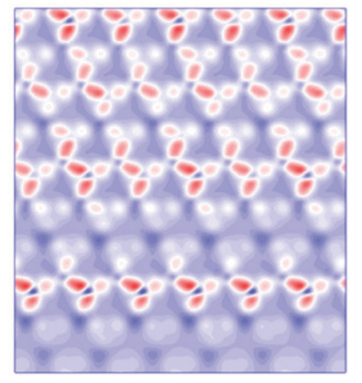

$\theta=2^{\circ}$

e

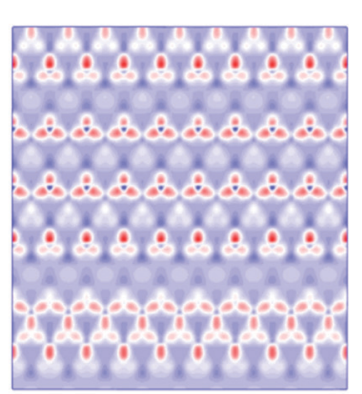

$\theta=2^{\circ}$
C

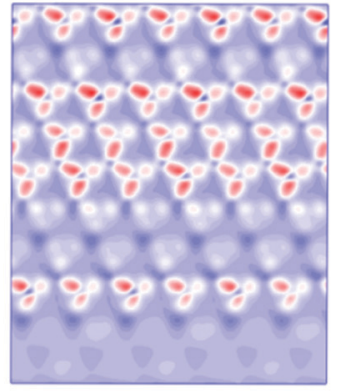

$\theta=4^{\circ}$

f

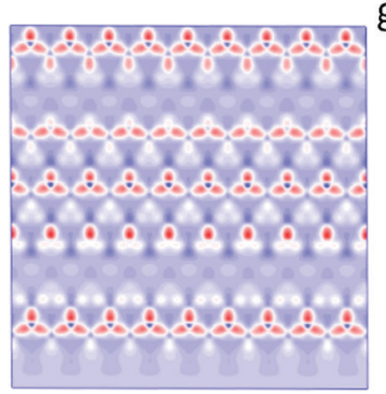

$\theta=4^{\circ}$ $\mathrm{h}$

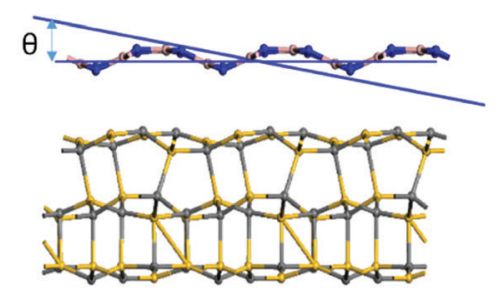

g

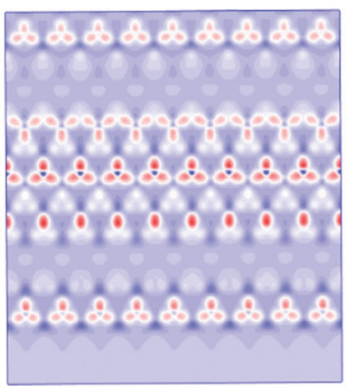

$\theta=6^{\circ}$

Fig. 3 Moire pattern of $\mathrm{BN} / \mathrm{SiC}-1$ with incident angles of (a) $\theta=0^{\circ}$, (b) $\theta=2^{\circ}$, (c) $\theta=4^{\circ}$, and BN/SiC-2 with incident angles of (d) $0 \AA$, (e) $\theta=2^{\circ}$, (f) $\theta=4^{\circ}$, (g) $\theta=6^{\circ}$; and (h) heterostructures with incident angle $\theta$ on the BN layer. 
wave-shaped geometry structure. The optimized heterostructures 1 and 2 are depicted in Table 1 . We calculate the total energy of the $\mathrm{BN}, \mathrm{SiC}$, and $\mathrm{BN} / \mathrm{SiC}$ stable structures, and the energy difference between them is calculated as the formation energy, ${ }^{29}$ using the equation $E_{\text {form }}=E_{\text {total }}^{\text {hetertructure }}-E_{\text {total }}^{\mathrm{SiC}}-E_{\text {total }}^{\mathrm{BN}}$, as presented in Table 2. The formation energies of the two $\mathrm{BN} / \mathrm{SiC}$ heterojunctions 1 and 2 are -1.26 and $-1.16 \mathrm{eV}$, respectively, and these values are within the range of our calculation accuracy. Therefore, we confirm that both structures are stable with $\mathrm{BN} / \mathrm{SiC}$ heterojunctions.

Additionally, we obtain the wave shape height $\mathrm{h}_{1}$ of the $\mathrm{BN}$ layer, as presented in Table 3 and Fig. 2. The height $(h)$ between the $\mathrm{BN}$ and SiC layers is the distance between the BN and SiC layers in the thickness direction of the slab. We calculate the different initial heights $(H)$ between the BN and SiC layers, and atomic distance of the BN layer, as shown in Table 4. The relaxation heights $h$ for the $\mathrm{BN}$ and $\mathrm{SiC}$ heterostructures are $2.43 \AA$ and $2.73 \AA$, respectively. The different initial heights change with the relaxation height $h$ of heterostructures 1 and 2 for small ranges $(\sim 0.1 \AA)$. After the relaxation, the distance between atom 1 and atom 2 of heterostructures 1 and 2 are within a small range $(\sim 0.02 \AA)$. This shows that the different heights between the $\mathrm{BN}$ and $\mathrm{SiC}$ layers will not change the wave shape structure of the heterostructures 1 and 2. A moiré structure is spatially periodic with a long-range period (in $\mathrm{nm}$ scale). Therefore, we have supercells in the $\mathrm{BN} / \mathrm{SiC}-1(3 \times 3)$ and $\mathrm{BN} / \mathrm{SiC}-2(3 \times 3)$ heterojunctions.

Previous studies have found that the moire pattern is obtained by rotating the angle of two atomic layers of $2 \mathrm{D}$ materials. ${ }^{18}$ However, we found that the $2 \mathrm{D}$ material with a single layer of the wave shape structure can obtain moiré patterns at different incident angles. Fig. 3 shows the moiré pattern of $\mathrm{BN} / \mathrm{SiC}-1$ heterostructures with different incident angles of the BN layer. Therefore, the optical effect of the double-layer material geometry structure can be realized by the single layer shape wave geometry material. Furthermore, the wave shape structure of the h-BN surface is obtained by treating with H-plasma. In the experiment, the h-BN flakes appear as bubbles on the h-BN surface after H-plasma treatment. ${ }^{30}$ Also, a large strain will be generated on the h-BN surface by gas treatment. Our results show that lattice strain $>5 \%$ will cause a moire pattern on the $\mathrm{BN} / \mathrm{SiC}$ heterojunctions. The results obtained in the experiment are consistent with our calculations.

\subsection{Band structure, work function, and density of states (DOS)}

We determine the band structures of the atomic monolayers of $\mathrm{BN}$ and SiC, whose band gaps are listed in Table 3 . It can be seen from Fig. 4 that the band gaps of the atomic layers of $\mathrm{BN}$ and $\mathrm{SiC}$ are 4.661 and $2.108 \mathrm{eV}$, respectively. The band gaps of the $\mathrm{BN} / \mathrm{SiC}$ van der Waals heterojunction structures 1 and 2 are 0.851 and $1.373 \mathrm{eV}$, respectively. The band gap of $\mathrm{BN}$ is reduced to approximately $0.7 \sim 1.4 \mathrm{eV}$. We find that the BN strain of structure $1(7.37 \%)$ is higher than that of structure $2(5.93 \%)$, whereas the band gap of structure $1(0.851 \mathrm{eV})$ is lesser than that of structure $2(1.373 \mathrm{eV})$. The results indicate that the strain of $\mathrm{BN} / \mathrm{SiC}$ increases, as the band gap decreases.
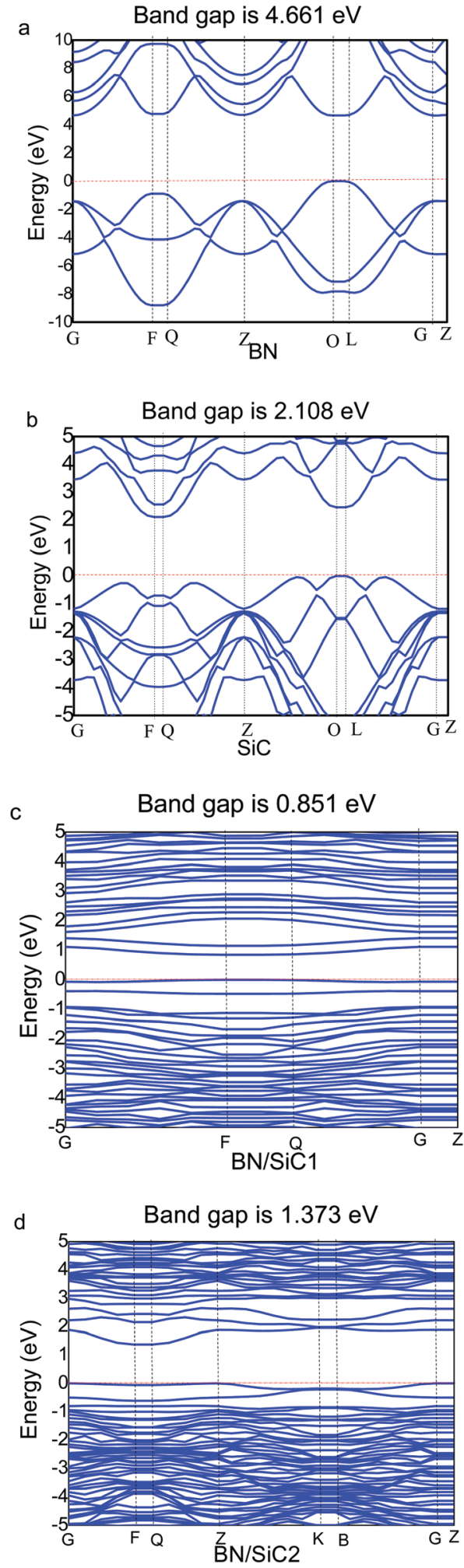

Fig. 4 Band structure of (a) BN, (b) $\mathrm{SiC}$, (c) BN/SiC-1, and (d) BN/SiC-2.

We calculate the work function of the $\mathrm{BN} / \mathrm{SiC}$ heterostructures, as presented in Table 3. The work function is the initial energy of the Fermi level and can be defined as the minimum energy required by the electrons to escape into a vacuum from 
the inside of the metal. In semiconductors, the energy at the bottom of the conduction band and valence band is typically lower than the minimum energy required for the electrons to escape from the metal. The work functions of BN/SiC structures 1 and 2 are 4.512 and $4.912 \mathrm{eV}$, respectively. A lower work function implies that electrons can easily escape the surface of the BN/SiC heterostructure. It can be seen from Table 3 that the work function of $\mathrm{BN} / \mathrm{SiC}$ structure 1 is smaller than that of structure 2, which implies that the electrons of $\mathrm{BN} / \mathrm{SiC}$ structure 1 can easily escape the surface than those of structure 2 .

We calculate the local density of states (LDOS) of the BN/SiC heterojunction, as depicted in Fig. 6. From Fig. 6a and b, we can see that the electronic distributions of the conduction band minimum (CBM) of the BN/SiC structures 1 and 2 are 0.851 and $1.373 \mathrm{eV}$, respectively. The electrons in the valence band maximum (VBM) are mainly distributed in Fermi surface $E_{\mathrm{F}}$. The LDOS is mainly contributed by the p orbital in the range from -2 to $2 \mathrm{eV}$; therefore, we compared the contribution of $\mathrm{C} 2 \mathrm{p}$, Si 2p, B 2p, and N 3p atoms to the band structure, as depicted in Fig. $6 \mathrm{c}$ and d. We found that the main orbital contribution to the CBM and VBM of the BN/SiC structures 1 and 2 originates from the Si $3 p$ and $C 2 p$ orbitals. The results indicate that the SiC substrate has a significant influence on the $\mathrm{BN}$ band structure in the $\mathrm{BN} / \mathrm{SiC}$ van der Waals heterojunction.

The band arrangement of the heterojunction relative to the potential of the hydrogen evolution reaction/oxygen evolution reaction (HER/OER) has an important influence on the photocatalytic decomposition of water. ${ }^{31}$ The energies of the hydrogen $\left(\mathrm{H}_{+} / \mathrm{H}_{2}\right)$ and oxygen evolution $\left(\mathrm{O}_{2} / \mathrm{H}_{2} \mathrm{O}\right)$ are -4.44 and $-5.67 \mathrm{eV}$, respectively. ${ }^{32}$ Fig. 5 compares the band edge positions of the $\mathrm{BN} / \mathrm{SiC}$ structures 1 and 2 of hydrogen evolution $\left(\mathrm{H}_{+} / \mathrm{H}_{2}\right)$ and oxygen evolution $\left(\mathrm{O}_{2} / \mathrm{H}_{2} \mathrm{O}\right)$. As seen in Fig. 6, the energies of the $\mathrm{BN} / \mathrm{SiC}-2$ structure of the CBM and VBM relative to the vacuum level are -3.539 and $-4.912 \mathrm{eV}$, respectively. Our results reveal that the $\mathrm{BN} / \mathrm{SiC}-2$ heterostructure could be a promising material for hydrogen evolution and optoelectronic devices.

\subsection{Chemical bonding states and deformation charge density}

From the BOLS-BC model, we derived the following formula for calculating the atomic energy density $E_{\mathrm{D}}\left(\mathrm{eV} \mathrm{nm}^{-3}\right)$ of a $2 \mathrm{D}$ heterojunction: $E_{\mathrm{D}}(z)=(1-\bar{\varepsilon})^{-(m+3)}$. The parameter $m$ is the

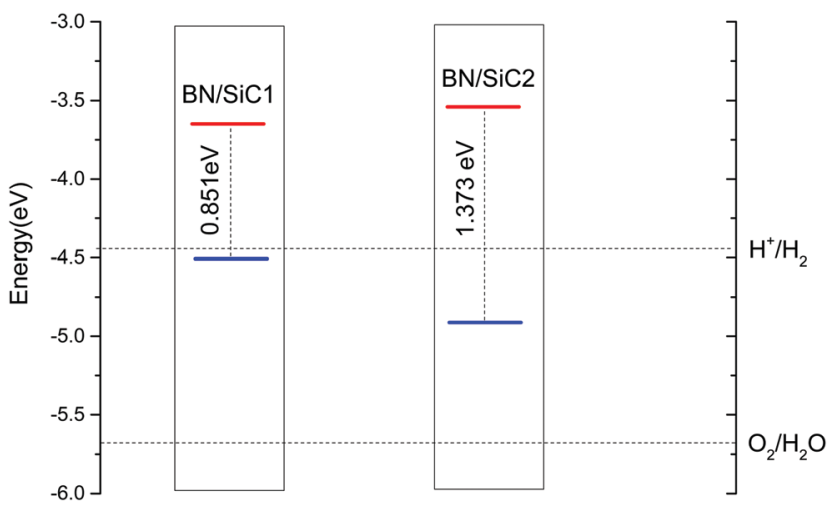

Fig. 5 The band edge alignment of $\mathrm{BN} / \mathrm{SiC}-1$ and $\mathrm{BN} / \mathrm{SiC}-2$ heterostructures indicator of the nature of the bond. The lattice strain $\bar{\varepsilon}$ of each atom determines the distribution of energy density; a high energy density causes a high lattice strain $\varepsilon$. We believe that the
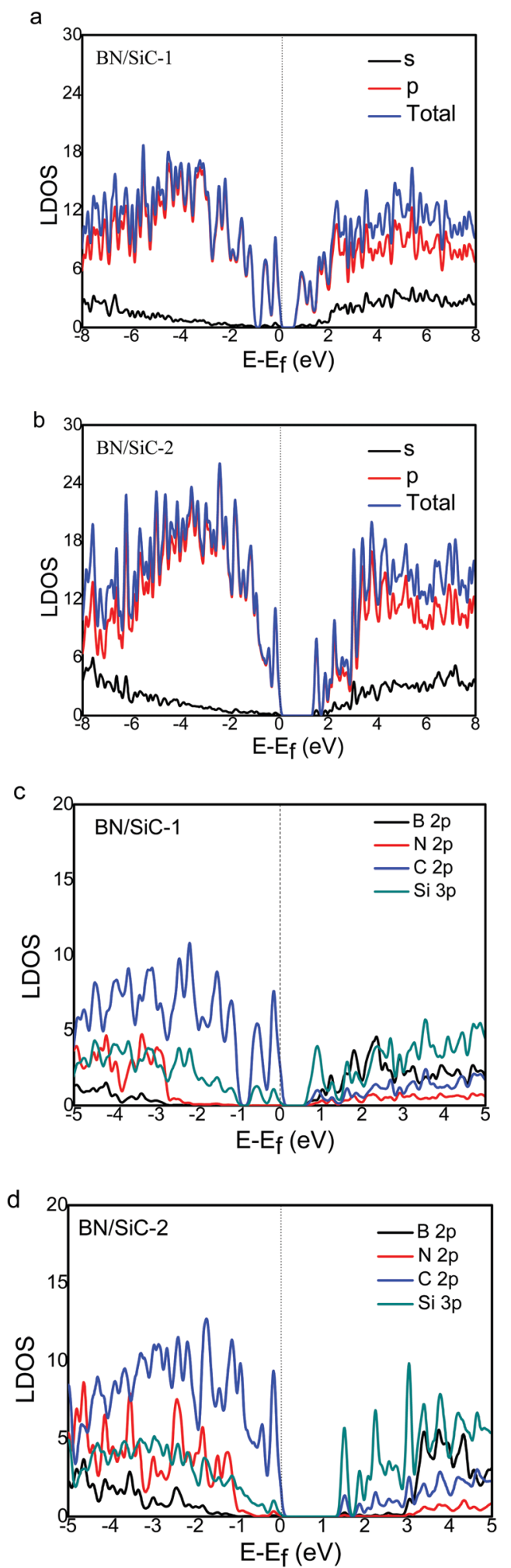

Fig. 6 The $\mathrm{s}$ - and p-orbital DOS of (a) BN/SiC-1 and (b) BN/SiC-2 heterostructures. The $\mathrm{B}, \mathrm{N}, \mathrm{C}$ and $\mathrm{Si}$ atoms DOS of (c) BN/SiC-1 and (d) $\mathrm{BN} / \mathrm{SiC}-2$ heterostructures. 
formation of $\mathrm{BN} / \mathrm{SiC}$ van der Waals heterostructures is primarily due to bond, electron, and charge contributions. Deformation charge density $\left(\mathrm{e} \AA^{-3}\right.$ ) is a tool used to study the electron transfer between atoms. In Fig. 7, we depict the electronic states of four chemical bonding states, namely, the antibonding, nonbonding, and bonding states and electron holes. ${ }^{33}$ The blue area represents the loss of electrons, and the red area represents the acquisition of electrons.

Antibonding states indicate the electron density and bond energy decrease in the region. Nonbonding states indicate that the electron density increases in the region, but the surrounding electron density does not overlap with the electron density of the other atoms. Bonding states indicate that the
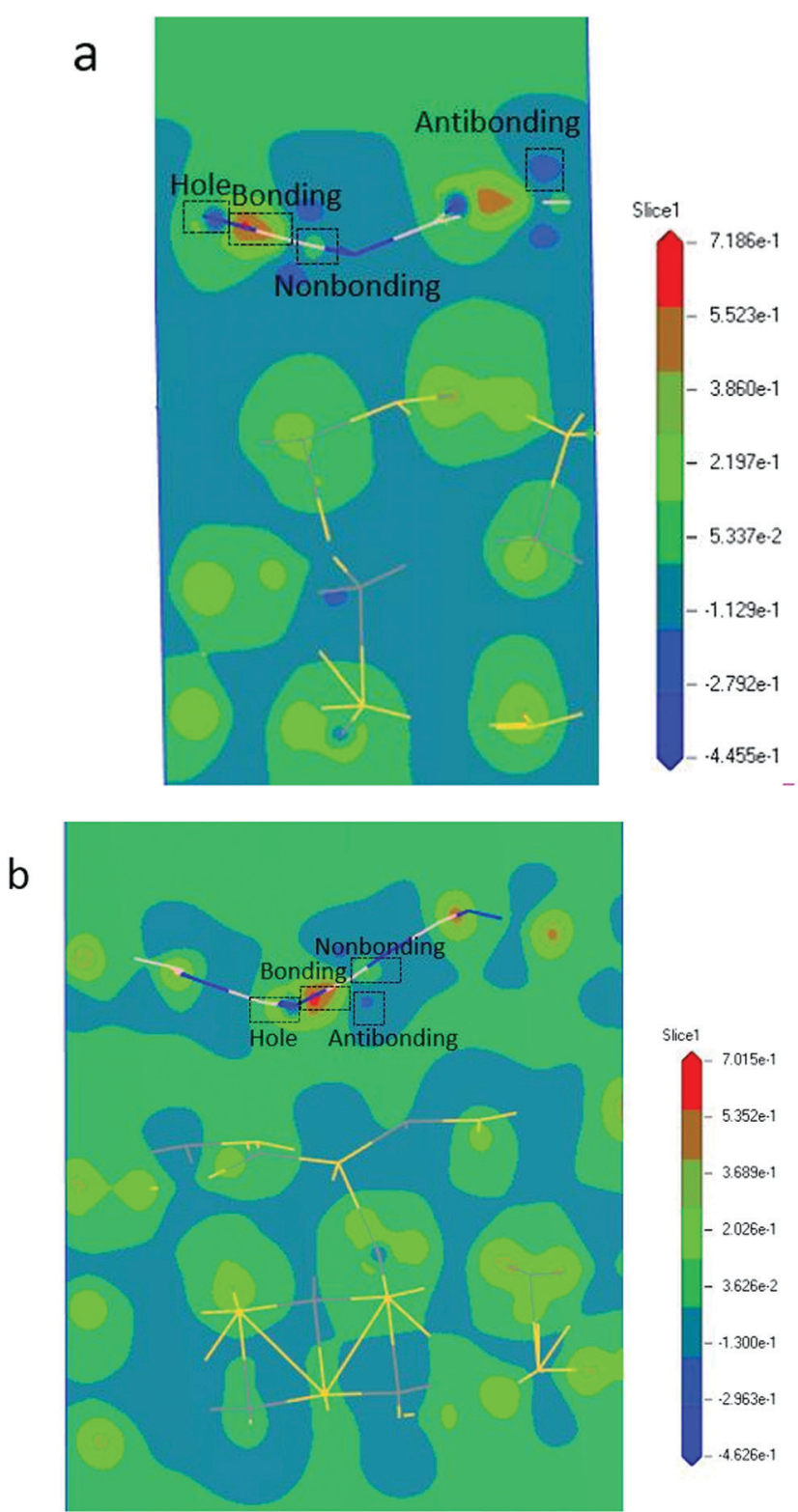

Fig. 7 Deformation charge density of (a) $\mathrm{BN} / \mathrm{SiC}-1$ and (b) BN/SiC-2 heterostructures resolved bonding states and electron features: nonbonding, antibonding, and bonding states, as well as electron holes.
Table 5 Chemical bonding states, deformation charge density $\Delta \rho_{i}(r)$, and potential functions $V_{\text {cry }}\left(r_{i}\right)$ of the van der Waals heterojunctions using the calculated BC $\left(\varepsilon_{0}=8.85 \times 10^{-12} \mathrm{C}^{2} \mathrm{~N}^{-1} \mathrm{~m}^{-2}, e=1.60 \times 10^{-19} \mathrm{C}\right)$

\begin{tabular}{lll}
\hline & $\begin{array}{l}\mathrm{BN} / \mathrm{SiC}-1 \\
(r=d / 2=1.445 / 2 \AA)\end{array}$ & $\left.\begin{array}{l}\mathrm{BN} / \mathrm{SiC}-2 \\
(r=d / 2=1.425 / 2\end{array}\right)$ \\
\hline$\Delta \rho_{\text {hole }}\left(r_{i}\right)\left(\mathrm{e} \AA^{-3}\right)$ & $-4.455 \times 10^{-1}$ & $-4.626 \times 10^{-1}$ \\
$\Delta \rho_{\text {bonding-electron }}\left(r_{i}\right)\left({\left.\mathrm{e} \AA^{-3}\right)}^{-3}\right.$ & $7.186 \times 10^{-1}$ & $7.015 \times 10^{-1}$ \\
$\Delta \rho_{\text {nonbonding-electron }}\left(r_{i}\right)\left(\mathrm{e} \AA^{-3}\right)$ & $2.197 \times 10^{-1}$ & $2.026 \times 10^{-1}$ \\
$\Delta \rho_{\text {antibonding-electron }}\left(r_{i}\right)\left(\mathrm{e}^{-3}\right)$ & $-2.792 \times 10^{-1}$ & $-2.963 \times 10^{-1}$ \\
$V_{\text {crybonding }}^{\text {nonbin }}\left(r_{i}\right)(\mathrm{eV})$ & -1.460 & -1.248 \\
$V_{\text {cry }}^{\text {bonding }}\left(r_{i}\right)(\mathrm{eV})$ & -4.775 & -4.322 \\
$V_{\text {cry }}^{\text {Antibonding }}\left(r_{i}\right)(\mathrm{eV})$ & 1.855 & 1.825
\end{tabular}

electron density increases in the region, but the electron density overlaps with the other atomic electron densities, indicating that the atom is in the bonding state. Electron holes indicate that the electron density decreases in the region, with a high concentration around the nucleus.

Furthermore, by defining the electronic states of the chemical bonding, we establish the expressions for the deformation charge density and potential function of the chemical bond states. The chemical bond potential function can be obtained by calculating the differential charge density. We used eqn (2) to calculate the potential functions of the antibonding, nonbonding, and bonding states. Comparing the potential functions, we obtain $V_{\text {cry }}^{\text {bonding }}(r)>V_{\text {cry }}^{\text {nonbonding }}(r)>V_{\text {cry }}^{\text {Antibonding }}(r)$. We also calculate the potential function of the atomic bonding states, as presented in Table 5. These results improve the theoretical reference for analyzing the quantitative bond properties of materials.

\section{Conclusions}

In this study, we investigated the geometric, energetic, electronic, and photocatalytic properties of $\mathrm{BN} / \mathrm{SiC}$ van der Waals heterostructures. A lattice strain $>5 \%$ results from the waveshaped geometry structure of the $\mathrm{BN} / \mathrm{SiC}$ heterojunctions. The wave shape can cause a moiré pattern with different incident angles. The BN/SiC van der Waals heterojunctions 1 and 2 will form moiré patterns at different incident angles, which can be applied in photoelectric detection technology. The BN/SiC van der Waals heterostructures 1 and 2 exhibit a band gap of 0.851 and $1.373 \mathrm{eV}$, respectively. The band arrangement shows that the $\mathrm{BN} / \mathrm{SiC}$ van der Waals heterojunction is a potential photoelectric material candidate. The DFT calculation and BOLS-BC model reveal the characteristics of the charge density and chemical bonds on the heterojunction's material surface. These results improve the theoretical reference for the analysis of the quantitative bond and charge properties of $2 \mathrm{D}$ heterojunction materials.

\section{Conflicts of interest}

There are no conflicts to declare. 


\section{Acknowledgements}

Financial support was provided by the National Natural Science Foundation of China (Grant No. 11947021) and the Research Program of Chongqing Municipal Education Commission (Grant No. KJQN201901421).

\section{References}

1 K. S. Novoselov, A. K. Geim, S. V. Morozov, D. Jiang, Y. Zhang, S. V. Dubonos, I. V. Grigorieva and A. A. Firsov, Science, 2004, 306, 666-669.

2 L. Li, Y. Yu, G. J. Ye, Q. Ge, X. Ou, H. Wu, D. Feng, X. H. Chen and Y. Zhang, Nat. Nanotechnol., 2014, 9, 372.

3 A. Splendiani, L. Sun, Y. Zhang, T. Li, J. Kim, C.-Y. Chim, G. Galli and F. Wang, Nano Lett., 2010, 10, 1271-1275.

4 M. Pumera and Z. Sofer, Adv. Mater., 2017, 29, 1605299.

5 K. S. Novoselov, D. Jiang, F. Schedin, T. J. Booth, V. V. Khotkevich, S. V. Morozov and A. K. Geim, Proc. Natl. Acad. Sci. U. S. A., 2005, 102, 10451-10453.

6 M. Bo, J. Li, C. Yao, Z. Huang, L. Li, C. Q. Sun and C. Peng, RSC Adv., 2019, 9, 9342-9347.

7 T. Hu and J. Hong, ACS Appl. Mater. Interfaces, 2015, 7, 23489-23495.

8 J. Qi, X. Qian, L. Qi, J. Feng, D. Shi and J. Li, Nano Lett., 2012, 12, 1224-1228.

9 Y. R. Tang, Y. Zhang and J. X. Cao, J. Appl. Phys., 2016, 119, 195303.

10 J. He, N. Jiao, C. Zhang, H. Xiao, X. Chen and L. Sun, J. Phys. Chem. C, 2014, 118, 8899-8906.

11 J. Wang, H. Shu, P. Liang, N. Wang, D. Cao and X. Chen, J. Phys. Chem. C, 2019, 123, 3861-3867.

12 D. S. Koda, F. Bechstedt, M. Marques and L. K. Teles, J. Phys. Chem. C, 2017, 121, 3862-3869.

13 K. Tang, W. Qi, Y. Li and T. Wang, J. Phys. Chem. C, 2018, 122, 7027-7032.

14 B. Sachs, T. O. Wehling, M. I. Katsnelson and A. I. Lichtenstein, Phys. Rev. B: Condens. Matter Mater. Phys., 2011, 84, 195414.

15 G. Chen, A. L. Sharpe, P. Gallagher, I. T. Rosen, E. J. Fox, L. Jiang, B. Lyu, H. Li, K. Watanabe, T. Taniguchi, J. Jung, Z. Shi, D. Goldhaber-Gordon, Y. Zhang and F. Wang, Nature, 2019, 572, 215-219.
16 M. M. van Wijk, A. Schuring, M. I. Katsnelson and A. Fasolino, Phys. Rev. Lett., 2014, 113, 135504.

17 C. Zhang, C.-P. Chuu, X. Ren, M.-Y. Li, L.-J. Li, C. Jin, M.-Y. Chou and C.-K. Shih, Sci. Adv., 2017, 3, e1601459.

18 M. R. Rosenberger, H.-J. Chuang, M. Phillips, V. P. Oleshko, K. M. McCreary, S. V. Sivaram, C. S. Hellberg and B. T. Jonker, ACS Nano, 2020, 14(4), 4550-4558.

19 Y. Cao, V. Fatemi, S. Fang, K. Watanabe, T. Taniguchi, E. Kaxiras and P. Jarillo-Herrero, Nature, 2018, 556, 43.

20 J. Kang, J. Li, S.-S. Li, J.-B. Xia and L.-W. Wang, Nano Lett., 2013, 13, 5485-5490.

21 S. K. Hämäläinen, M. P. Boneschanscher, P. H. Jacobse, I. Swart, K. Pussi, W. Moritz, J. Lahtinen, P. Liljeroth and J. Sainio, Phys. Rev. B: Condens. Matter Mater. Phys., 2013, 88, 201406.

22 M. Sicot, S. Bouvron, O. Zander, U. Rüdiger, Y. S. Dedkov and M. Fonin, Appl. Phys. Lett., 2010, 96, 093115.

23 M. Yankowitz, J. Jung, E. Laksono, N. Leconte, B. L. Chittari, K. Watanabe, T. Taniguchi, S. Adam, D. Graf and C. R. Dean, Nature, 2018, 557, 404-408.

24 J. R. Wallbank, M. Mucha-Kruczyński, X. Chen and V. I. Fal'ko, Ann. Phys., 2015, 527, 359-376.

25 J. Heyd, G. E. Scuseria and M. Ernzerhof, J. Chem. Phys., 2003, 118, 8207-8215.

26 M. Bo, Y. Wang, Y. Huang, Y. Liu, C. Li and C. Q. Sun, Appl. Surf. Sci., 2015, 325, 33-38.

27 C. Q. Sun, Relaxation of the Chemical Bond, Heidelberg, New York, Dordrecht, London, Singapore, 2014.

28 M. Bo, Y. Guo, Y. Wang, Y. Liu, C. Peng, C. Q. Sun and Y. Huang, Phys. Chem. Chem. Phys., 2016, 18, 13280-13286.

29 J. Liao, B. Sa, J. Zhou, R. Ahuja and Z. Sun, J. Phys. Chem. C, 2014, 118, 17594-17599.

30 L. He, H. Wang, L. Chen, X. Wang, H. Xie, C. Jiang, C. Li, K. Elibol, J. Meyer, K. Watanabe, T. Taniguchi, Z. Wu, W. Wang, Z. Ni, X. Miao, C. Zhang, D. Zhang, H. Wang and X. Xie, Nat. Commun., 2019, 10, 2815.

31 S. Tang, J. Yu and L. Liu, Phys. Chem. Chem. Phys., 2013, 15, 5067-5077.

32 G. Wang, L. Zhang, Y. Li, W. Zhao, A. Kuang, Y. Li, L. Xia, Y. Li and S. Xiao, J. Phys. D: Appl. Phys., 2019, 53, 015104.

33 M. Bo, L. Li, Y. Guo, C. Yao, C. Peng and C. Q. Sun, Appl. Surf. Sci., 2018, 427, 1182-1188. 